\title{
Análisis crítico del discurso de profesores de matemáticas y sus estudiantes: subjetividades y saberes en aulas heterogéneas
}

\author{
Critical analysis of discourses of mathematics teachers and their students: \\ subjectivities and knowledge in heterogeneous classrooms
}

\author{
Fabián Andrés Inostroza Inostroza \\ Pontificia Universidad Católica de Chile, Facultad de Educación \\ Programa de Magíster en Educación \\ Telf.: (56) 229693047. Correo electrónico: fainostr@uc.cl
}

\begin{abstract}
RESUMEN
En el presente artículo se realiza un análisis crítico del discurso de docentes y alumnos respecto a la noción de estudiante de buen rendimiento académico en matemáticas (EBRAM) y los saberes de las matemáticas que este educando debe dar cuenta. El estudio se enmarca dentro de una tradición cualitativa en investigación, empleando al postestructuralismo como enfoque teórico/metodológico y las nociones de discurso y gubernamentalidad construidas por Michel Foucault. Los resultados evidencian que la noción de EBRAM corresponde a la construcción de un estudiante que posee una habilidad matemática innata, que es predominantemente un sujeto racional, que posee autorregulación sobre sus conductas y que pertenece a una familia bien constituida. Por último, los efectos de esta construcción normativa en los estudiantes categorizados como EBRAM y como no EBRAM, señalan que estos sujetos escolarizados tienden a performar la etiqueta discursiva que les ha sido asignada dentro del contexto educativo estudiado.

Palabras clave: análisis crítico del discurso, estudiante de buen rendimiento académico en matemáticas, gubernamentalidad.
\end{abstract}

\section{ABSTRACT}

This article aims to make a critical discourse analysis of teachers and students regarding the notion of successful student in mathematics and the mathematical knowledge that such students must give account. The study is part of a qualitative research tradition using poststructuralism as a theoretical / methodological approach and notions of discourse and governmentality built by Michel Foucault. The research results show that the notion of successful student in mathematics is the construction of a student who has an innate mathematical ability, who is a rational subject, who has self-regulation on his/her behavior and, who belongs to a well-established family. Finally, the effects of the creation of this category in students categorized in this way, note that these subjects tend to perform the discursive label assigned to them within the educational context studied.

Key words: critical discourse analysis, successful student in mathematics, governmentality. 


\section{INTRODUCCIÓN}

En este artículo se pretende analizar críticamente los discursos de los docentes de matemática y de sus alumnos en torno a la noción del estudiante de buen rendimiento académico en matemática (de aquí en adelante EBRAM) y de los saberes que este debería dar cuenta. Para ello se emplea el análisis crítico del discurso, siguiendo los aportes y lineamientos que Foucault (2013) propone en este ámbito.

El propósito principal de esta investigación es describir y problematizar la norma construida desde los discursos de los docentes de matemática y sus estudiantes respecto al EBRAM y los saberes de la matemática escolar ${ }^{1}$ que este debe dar cuenta.

En este sentido la descripción y problematización de la norma permitirá visibilizar cómo estas construcciones generan una tensión permanente en los sistemas educativos, en donde tanto profesores como estudiantes son instados por medio de mecanismos y tecnologías de saber/poder como lo son las políticas públicas en educación y el currículum oficial, a modificar su subjetividad alejándose o acercándose de estas construcciones normativas de carácter ficticio, que son a su vez impuestas como forma de asegurarse el gobierno del estudiante y, por consiguiente, la construcción y gestión de su "yo" (Rose, 1999).

En cuanto a las interrogantes que orientan este estudio y que formarán parte fundamental del análisis de los discursos de los participantes de esta investigación, estas corresponden a: ¿Cuál es la noción de EBRAM que está presente en las narrativas de los docentes y estudiantes? ¿Cuáles son los saberes de las matemáticas que son legitimados en los discursos de los docentes? ¿Qué efecto(s) tiene la construcción discursiva del EBRAM en aquellos estudiantes que se acercan/alejan de esta noción?

\section{ANTECEDENTES Y PROBLEMATIZACIÓN}

Actualmente, la matemática escolar se ha posicionado como una de las disciplinas claves dentro del currículum escolar, debido a que el aprendizaje de esta asignatura se asocia al desarrollo de competencias y habilidades que tendrán una repercusión positiva en la producción de capital humano avanzado, el cual será empleado posteriormente en el mundo laboral (BID, 2010).

Esta visión respecto a la matemática escolar es compartida y promovida a nivel internacional por organismos intergubernamentales vinculados con la educación como el Banco Interamericano de Desarrollo (BID), la Organización para la Cooperación y el Desarrollo Económico (OCDE) y la Organización de las Naciones Unidas para la Educación, Ciencia y Cultura o UNESCO (Kanes, Morgan y Tsatsaroni, 2014).

\footnotetext{
En esta investigación se hará una distinción entre las matemáticas y la matemática escolar con el fin de precisar el uso que se le está dando a cada una de ellas. Por las matemáticas se entenderá: "como una rama del saber científico establecido, con sólidos criterios de verdad y con comunidades científicas internacionalmente robustas" (Cantoral, Montiel y ReyesGasperini, 2014, p. 103). En tanto la matemática escolar será entendida como: "un subproducto de las matemáticas derivado de los procesos de transposición hacia el ámbito escolar y por así decirlo, una escenificación de las matemáticas en el aula" (Cantoral et al., 2014, p. 103).
} 
Son estas mismas agencias internacionales las que han creado sistemas de evaluación estandarizados guiados por una lógica denominada como las Reformas Basadas en Estándares (RBE), enfoque que supone que la experiencia escolar de los estudiantes puede ser reducida a un puntaje en un test (Casassus, 2010). Bajo esta misma visión se crean mecanismos para medir la calidad de la educación, por medio de pruebas internacionales tales como el Programa Internacional para la Evaluación de Aprendizajes (PISA, por sus siglas en inglés), el Estudio Internacional de Tendencias en Matemáticas y Ciencias (TIMSS, por sus siglas en inglés), el Estudio Internacional del Progreso en Competencia Lectora (PIRLS, por sus siglas en inglés), entre otros (Popkewitz, 2004).

De acuerdo a las últimas mediciones en evaluaciones estandarizadas a nivel internacional, se ha informado que los resultados obtenidos por los estudiantes de Latinoamérica en matemáticas han sido bajos o elementales. Esto de acuerdo a los informes más recientes emanados por TIMSS y PISA (Agencia de la Calidad de la Educación, 2014).

La misma tendencia se replica a nivel de la educación chilena, de acuerdo a lo informado en la última medición realizada en matemática escolar por la prueba SIMCE (Sistema de Medición de la Calidad de la Educación). Los desempeños obtenidos por los estudiantes chilenos en los últimos años en este test han sido catalogados dentro de los rangos de elemental e insatisfactorio (Agencia de la Calidad de la Educación, 2014).

Uno de los aspectos que más ha causado impacto en la investigación educativa en este ámbito es la significativa brecha de los rendimientos en las pruebas de matemática escolar entre los colegios de dependencia particular pagada y los municipalizados (Aravena y Caamaño, 2007; Donoso y Hawes, 2002). De acuerdo a la última aplicación de la evaluación SIMCE (2013) pesquisada, la diferencia de puntajes en la prueba de matemática escolar de $8^{\circ}$ año de educación básica entre los colegios particulares y los municipales fue de 53 puntos, lo que implica que un porcentaje cercano al $45 \%$ de los alumnos que asisten a los establecimientos municipalizados no dan cuenta de los aprendizajes básicos para su nivel educativo, esto de acuerdo a las Bases Curriculares vigentes (Agencia de la Calidad de la Educación, 2014).

Ante un escenario como el descrito con anterioridad, se comienza a naturalizar una tesis respecto a un presunto déficit en los estudiantes (Peña, 2013). De hecho, existe un número importante de literatura que circula, produce y reproduce dicha creencia, según la cual la problemática de los bajos resultados de los alumnos que asisten a escuelas municipales en pruebas estandarizadas estaría relacionado con un déficit cultural vinculado con las diferencias existentes entre los códigos lingüísticos de la escuela y el hogar, o también debido a situaciones de precariedad (en términos socioeconómicos, materiales, entre otros) y de riesgo social de los contextos en los que viven los educandos que asisten a estos centros educativos (Bellei et al., 2004; Castro et al., 2013).

En la misma línea de análisis, existen investigaciones que señalan que esta forma de mitificar o validar la tesis del déficit impacta profundamente en las creencias, actitudes y expectativas que tienen los profesores hacia los estudiantes que asisten a estos establecimientos educacionales (Aravena y Caamaño, 2007; Infante, Matus y Vizcarra, 2011; Román, 2011).

Debido a la circulación y legitimación de este tipo de discursos dentro de la investigación hasta el momento producida en este ámbito, es que se hace necesario problematizar la noción de "norma" o EBRAM y de los saberes de la matemática escolar que este estudiante debe dar cuenta, de tal forma que por medio de su descripción y su 
consecuente problematización, este ejercicio analítico permita reflexionar a los agentes educativos involucrados respecto a la naturaleza del EBRAM como una noción conceptual más que como una categoría preexistente. Además, es necesario notar que esta norma emerge dentro de un contexto histórico, social, cultural e ideológico, marcado por esquemas regulatorios planteados desde políticas neoliberales aplicadas al ámbito educativo, lo que tiene implicancias en la producción de subjetividades dentro del aula (Falabella, 2014; Infante et al., 2011; Popkewitz y Brennan, 2000; Veiga-Neto, 2010).

A continuación se realizará una breve descripción de los dos núcleos conceptuales fundamentales para esta investigación. Estos constituyen, por un lado, al objeto de estudio que corresponde a la noción de EBRAM, y por otro lado se definirán los saberes de la matemática escolar que son considerados como legítimos para los docentes en este contexto educativo. Luego de ser definidos estos conceptos, será en torno a ellos que se realizará el análisis crítico del discurso (de aquí en adelante, ACD), enfoque de carácter teórico metodológico empleado con el fin de proporcionar respuestas a las interrogantes ya enunciadas con anterioridad.

\section{MARCO CONCEPTUAL}

\subsection{EL ESTUDIANTE DE BUEN RENDIMIENTO ACADÉMICO EN MATEMÁTICAS (EBRAM)}

Para los fines de esta investigación, la descripción de lo que corresponde un EBRAM se realizará siguiendo los lineamientos que propone el currículum nacional a través de las Bases Curriculares en Educación Matemática del año 2012 para los niveles de $5^{\circ}$ y $6^{\circ}$ año básico y los ajustes curriculares del año 2009 para los niveles de $7^{\circ}$ y $8^{\circ}$ año de educación básica, niveles educativos en los cuales se desarrolló este estudio. Además será descrita esta noción incluyendo aquellas investigaciones cuyos enfoques tienen como punto de partida la problematización y el cuestionamiento de esta construcción normativa realizada en y a través las políticas educativas vinculadas a la norma y la diferencia en educación.

Para las Bases Curriculares en Educación Matemática (2012) se considera que es fundamental durante la educación básica que un estudiante pueda ser capaz de: "Comprender y utilizar conceptos y procedimientos matemáticos básicos, relativos a números y formas geométricas, en la resolución de problemas cotidianos y apreciar el aporte de la matemática para entender y actuar en el mundo" (MINEDUC, 2012, p. 15).

Bajo esta lógica queda en evidencia que el perfil de egreso que se pretende para un alumno "norma" está relacionado con el dar cuenta de los contenidos mínimos (saberes de la matemática escolar) asociados a un uso instrumental de esta asignatura escolar (Kanes et al., 2014; Popkewitz, 2013; Skovsmose, Alro y Valero, 2008). Lo anterior implica que estos saberes, al legitimarse como la norma o el aprendizaje normativo, naturalizan el hecho de que todos los estudiantes sin excepción deben obligatoriamente demostrar estos conocimientos para poder egresar y corresponder a un buen estudiante en matemáticas (Knijnik, 2007).

Si un estudiante no es capaz de lograr lo anteriormente mencionado, el alumno se encontrará emplazado fuera de la norma esperada. Estos supuestos respecto al educando norma refuerzan las teorías de desarrollo psicológico y cognitivo de los alumnos, modelos 
teóricos que asumen como base niveles de desarrollo normales, estructuras estáticas y fijas, en las cuales los niños que escapan de los parámetros determinados a priori son considerados como "deficitarios" o con "dificultades de aprendizaje" (Besley, 2009 Cit. en Infante et al., 2011; Walkerdine, 1997 Cit. en Llewelyn, 2012).

Por otra parte, existen enfoques que buscan problematizar esta noción de norma respecto del buen estudiante inscrita en las políticas educativas chilenas y en el currículum nacional. Se ha determinado, por medio de estas investigaciones, que el alumno promedio o norma al que se aspira correspondería, siguiendo a Infante (2010) a "(un estudiante) con un buen rendimiento académico acorde a los estándares educacionales chilenos (currículum escolar), nivel socioeconómico medio-alto, y nacido dentro de los límites geográficos que determina el país" (p. 289).

La descripción del buen estudiante en términos académicos expuesta en la cita anterior se constituye problemática dado que caracteriza a este alumno de manera restringida, estática y esencialista. En estos términos, se tendería a entender al buen estudiante relevando solo atributos asociados a su cognición (entendida como inteligencia) y su capacidad para aprender un currículum prescrito y una asignatura particular (Infante, 2010; Matus, 2005). Esto determina discursivamente al universo de los estudiantes que asisten a las escuelas chilenas, quienes no necesariamente se acercan a esta construcción conceptual debido a que sus subjetividades no pueden ser reducidas a priori a un estándar construido de manera teórica (Llewelyn, 2012; Popkewitz, 2004; Youdell, 2010).

En síntesis, de acuerdo a lo expuesto con anterioridad el EBRAM ha sido definido desde diversos enfoques, los cuales relevan de entre sus atributos fundamentales la racionalidad y el cumplimiento de ciertos estándares de desarrollo normal asociados a logros en el aprendizaje de los saberes de la matemática escolar en un nivel de educación formal determinado.

\subsection{SABERES DE LA MATEMÁTICA ESCOLAR}

Para la conceptualización de estos saberes se emplearán los contenidos que proponen las Bases Curriculares de Educación Matemática del año 2012 y los Ajustes Curriculares del año 2009, respectivamente, ambos documentos producidos y emanados por el MINEDUC.

De acuerdo a las Bases Curriculares del año 2012, los saberes pretendidos en los niveles $5^{\circ}$ y $6^{\circ}$ año de educación básica corresponden a: el concepto de número, las operaciones básicas de la aritmética (adición, sustracción, multiplicación y división), patrones y equivalencias, unidades de medidas convencionales (metro, kilogramo y litro), lectura e interpretación de información contenida en gráficos y tablas, conceptos geométricos relativos a objetos geométricos de dos dimensiones y de tres dimensiones (MINEDUC, 2012).

Respecto a los niveles correspondientes a $7^{\circ}$ y $8^{\circ}$ año de educación básica, los saberes de la matemática escolar que han sido definidos por medio de los Ajustes Curriculares del año 2009 y que deben demostrar los estudiantes corresponden a: los distintos conjuntos numéricos, las operaciones aritméticas básicas aplicadas a cada uno de los conjuntos numéricos, las potencias, las relaciones proporcionales, el lenguaje algebraico, las ecuaciones e inecuaciones, las características y propiedades de figuras y cuerpos geométricos, el área y perímetro de figuras y cuerpos geométricos, la muestra y población en estadísticas, los experimentos aleatorios y los modelos probabilísticos (MINEDUC, 2009). 


\section{METODOLOGÍA}

\subsection{DISEÑO}

El diseño metodológico que se utilizó para analizar los discursos de los docentes y estudiantes se inserta dentro de una tradición cualitativa de investigación (Creswell, 2007), y se consideró como metodología el Análisis crítico del discurso (ACD) desde una perspectiva postestructural empleando los aportes que el autor Michel Foucault (2013) propone en este ámbito. En este sentido, las nociones de discurso, relaciones de saber/ poder y la constitución de las subjetividades fueron centrales en el ejercicio analítico que se desarrolló en torno a las narrativas de docentes y alumnos.

Como instrumento de recopilación de información se empleó la entrevista en profundidad, ya que esta técnica permite encontrar lo que es significativo o importante para los sujetos desde sus perspectivas, sus interpretaciones y el modo en el cual ellos ven y experimentan su propio mundo (Ruiz, 2003). Además, este instrumento considera a los sujetos (docentes y estudiantes) como actores sociales que están insertos en un contexto, por tanto este espacio influye en la constitución de las subjetividades de los participantes de esta investigación (Foucault, 2013; Infante et al., 2011).

Así también, esta técnica asume que los sujetos entrevistados son capaces de dar sentido al mundo que habitan de acuerdo a los repertorios interpretativos que les son disponibles en un momento dado, para una sociedad y cultura determinada (Infante et al., 2011). Adicionalmente, este instrumento permitió obtener narrativas con la profundidad y densidad de la información necesarias para evidenciar coherencias y contradicciones en las formaciones discursivas relacionadas con el objeto de estudio que persigue esta investigación (Canales, 2012).

\subsection{PARTICIPANTES}

Para esta investigación se seleccionó una escuela de dependencia municipal de la comuna de Santiago Centro, en la cual se desarrolla el Proyecto FONDECYT N ${ }^{\circ} 1130616$, que es el marco desde donde surge este estudio. Se escogió dentro de los docentes del establecimiento a profesores de educación básica con mención en matemática que ejercen en el segundo ciclo de educación básica y que imparten actualmente esta asignatura en el colegio.

Se les solicitó a cada uno de estos docentes que escogieran, uno o dos alumnos para ser entrevistados. Además, se les explicó que debían seleccionar a un niño o joven que fuera un buen estudiante en matemática escolar y a otro que no lo fuera, de acuerdo a su criterio. Adicionalmente, una de las peticiones realizada a los educadores en la elección de los sujetos escolares fue la de escoger a sus estudiantes, tratando de resguardar que los jóvenes seleccionados fueran preferentemente de distinto género, edad, etnia, nacionalidad, etc. Finalmente, los educandos que fueron entrevistados corresponden a dos estudiantes de género masculino y dos de género femenino, los que se distribuyeron de la misma forma respecto al criterio del estudiante con buen rendimiento o bajo desempeño en matemática escolar. La muestra consta, finalmente, de 3 profesores y 4 estudiantes, con un total de 7 participantes. 


\subsection{PROCEDIMIENTOS}

Se construyeron dos tipos de entrevistas en profundidad: una para ser aplicada a los docentes y otra para los estudiantes. Para la elaboración de estas, se diseñaron dos guiones distintos. En el caso del guion creado para orientar la entrevista realizada a los educadores, este abordó temáticas vinculadas con sus experiencias como profesores de matemática escolar en el establecimiento, la forma en la cual caracterizan al EBRAM, los saberes de las matemáticas que favorecen en clases, la metodología empleada para enseñar dichos saberes, entre otros. Respecto al guion que sirvió de referencia para la entrevista aplicada a los alumnos, este trató temáticas relacionadas con el gusto o preferencia por la matemática escolar, la forma de entenderse a sí mismos como un EBRAM $-\mathrm{o}$, por el contrario, razonarse como un estudiante de bajo desempeño académico en esta asignatura- la manera en la cual el profesor organizaba la clase, si es que los docentes consideraban los saberes de las matemáticas que sus alumnos emplean en contextos distintos a la escuela, entre otros temas relacionados con las temáticas de interés para esta investigación.

Las entrevistas fueron realizadas en una única instancia a los 3 docentes por separado, cada una de estas tuvo una duración promedio de 1 hora y 30 minutos por educador; en el caso de los estudiantes, estas tuvieron una extensión de 45 minutos aproximadamente cada una. Las entrevistas fueron llevadas a cabo en las dependencias del establecimiento, dentro de la jornada escolar, procurando no interrumpir las actividades lectivas de los docentes y las de aprendizaje de los estudiantes que participaron en este estudio. Se consideró que en coherencia con el alcance de este estudio la cantidad de participantes se encuentra en concordancia con la densidad y complejidad de los discursos que se quieren analizar, por tanto, una muestra más amplia se considerará para una investigación posterior.

\section{HERRAMIENTAS TEÓRICAS METODOLÓGICAS}

Para analizar los discursos de los docentes y estudiantes se empleó un análisis crítico del discurso (ACD) de orientación foucaultiana. El ACD permite realizar una lectura deconstructiva que busca problematizar y cuestionar supuestos, creencias, interpretaciones de la realidad, saberes, cosmovisiones, etc., que en un tiempo y contexto determinado son considerados bajo ciertas reglas como verdades incuestionables (Infante et al., 2011). En particular, el ACD que se realizó en esta investigación busca describir (en el sentido foucaultiano) las formaciones discursivas en las cuales estén presentes la noción de EBRAM y los saberes de la matemática escolar que este debe dar cuenta.

Para llevar a cabo el análisis, la noción de discurso cobra una relevancia fundamental. Desde la conceptualización foucaultiana el discurso corresponde a: "un sistema de poder/conocimiento o prácticas que son creados históricamente y que son culturalmente localizados" (Infante et al., 2011, p. 145).

Debido a lo ya señalado, se torna relevante considerar al discurso desde esta perspectiva como un elemento central del ejercicio analítico. En efecto, razonar esta noción como una práctica social situada implica que el discurso emerge, circula y se (re)produce bajo determinadas condiciones históricas, culturales y sociales, que hacen que para un grupo humano determinado ciertos discursos sean considerados como verdades incuestionables (Canales, 2012; Espinoza, 2012; Hernández, 2010; Infante et al., 2011). Más aún, el 
discurso tiene la potencialidad de constituir los objetos que enuncia. En otras palabras, este concepto es crucial en la formación de subjetividades y de comportamientos, ya que es este quien define, en un espacio y tiempo determinado, lo que es deseable apropiado y, en definitiva, lo que es considerado como normal en términos de la construcción del yo (Ball, 1997; Espinoza, 2012; Infante et al., 2011; Jäger, 2008; Larrosa, 1995; Popkewitz y Brennan, 2000).

Además de relevar la importancia de la noción de discurso que se empleó en este estudio, también se hizo necesario elaborar una serie de herramientas de análisis para realizar el ACD. Para dicho fin se construyó una caja de herramientas foucaultianas Espinoza (2012).

Para realizar el ACD se diseñaron matrices en las cuales se organizaron fragmentos de las narrativas entendidas como discursos de docentes y estudiantes. Los componentes que incluyen las matrices responden, en parte, a las cuatro dimensiones que constituyen a las formaciones discursivas, de acuerdo a lo propuesto por Foucault (2013) en su obra La Arqueología del Saber. Estos componentes corresponden a la formación de: objetos, modalidades de enunciación, conceptos y elecciones estratégicas.

De entre estas cuatro dimensiones se han considerado para esta investigación solo dos: la formación de objetos, vinculada a la primera interrogante que hace mención a la construcción discursiva del EBRAM; y las elecciones de estrategias relacionadas con la segunda interrogante que hace alusión a los saberes de las matemáticas que son legitimados por los docentes. Así también, se ha hecho necesario incluir a la gubernamentalidad para dar respuesta a la tercera pregunta orientadora de este estudio, la que está asociada con la relación entre los discursos de los docentes y sus estudiantes y la constitución de subjetividades de los sujetos escolares presentes en esta institución educativa.

Para el caso de la formación de objetos, es importante señalar que el discurso emerge dentro un entramado de relaciones sociales, históricas e institucionales concretas, que para el caso de este estudio corresponderá a la institución denominada escuela (Espinoza, 2012; Popkewitz y Brennan, 2000). Lo que implica en esta investigación considerar cada una de las dimensiones señaladas con anterioridad en la emergencia del objeto de estudio o EBRAM.

En cuanto a la formación de estrategias, esta se entenderá como el proceso de inclusión y exclusión de ciertos enunciados dentro de una misma formación discursiva. Esto implica evidenciar las inclusiones exclusiones teóricas que se realizaron a nivel discursivo para que los docentes de matemática de esta institución, en este momento histórico, definieran y legitimaran ciertos saberes de las matemáticas por sobre otros (Canales, 2012; Espinoza, 2012).

Tal como se señaló anteriormente, se decidió incluir dentro de las matrices de análisis la gubernamentalidad. Esta noción se incorporó debido a que es fundamental para esta investigación analizar las relaciones existentes entre los discursos docentes y la constitución de las subjetividades de los estudiantes. En este sentido, Infante et al. (2011) proponen al respecto: "El concepto de gubernamentalidad permite reflexionar sobre la relación entre el poder/conocimiento y la construcción de subjetividades" (p. 4).

De manera más particular, la noción de gubernamentalidad permitirá establecer el vínculo existente entre los discursos docentes y sus estudiantes y la forma en la cual se orienta o guía la conducta, el comportamiento y, en definitiva, la construcción del yo de los educandos, de manera tal que sea posible visibilizar la forma en la cual los discursos de los participantes de esta investigación tienden lograr acercar o alejar a los estudiantes de la norma construida. 


\section{ANÁLISIS DE LAS NARRATIVAS}

\subsection{LA EMERGENCIA DEL OBJETO DE ESTUDIO: LA CONSTRUCCIÓN DISCURSIVA DEL EBRAM}

La construcción de EBRAM elaborada por los sujetos docentes entrevistados está constituida por cuatro atributos de acuerdo a las narrativas analizadas. Estos corresponden a: una habilidad matemática de naturaleza innata, la racionalidad, autorregulación y la pertenencia a una familia "bien constituida".

Respecto al primer atributo mencionado, en las narrativas de los profesores se evidencia que es indispensable que un EBRAM posea una habilidad matemática superior, la cual le permitiría distinguirse de entre sus pares. De hecho, se propone que en cada curso la presencia de esta característica es muy baja, por tanto, son solo algunos estudiantes privilegiados quienes poseen dicha habilidad. De esta forma confirma lo anteriormente expuesto un educador: "Pero siempre dentro de todo el espectro educativo hay pocos niños que tienen una habilidad matemática que la traen, que como se dice, es innata y eso los hace mejores en matemática". (Profesor 1, p. 7).

Al poseer esta habilidad matemática, el EBRAM se ve impelido a ir más allá de lo solicitado por el docente, generando un tipo de respuesta a las situaciones problemáticas en matemáticas distintas al del resto de sus pares. Se afirma que esta habilidad le permitiría al EBRAM generar un razonamiento matemático más elaborado que el de sus compañeros:

"Lo que pasa es que su potencial no puede ser el mismo (...) yo creo que los estudiantes que van por el lado de la matemática son aquellos que ven más allá, que tienen la habilidad, que no solamente se quedan en el cálculo, que te pueden dar otro nivel de respuestas, mucho más avanzadas". (Profesor 3, p. 9).

El segundo atributo mencionado y vinculado con la habilidad matemática innata es la racionalidad. Para los docentes, el EBRAM es, ante todo, un estudiante racional (sujeto cartesiano) que emplea la lógica matemática, la que le permite elaborar una cadena de razonamiento para deducir de entre un conjunto de datos una respuesta satisfactoria a una situación problemática en matemática escolar (Cantoral, Montiel, Reyes-Gasperini, 2014). Como un elemento adicional se señala en las narrativas de los profesores, la importancia de producir respuestas que exceden a la correcta aplicación de algoritmos. Bajo estos términos lo expone un docente:

"Porque este tipo de estudiantes debe utilizar la lógica y crear, crear una digámoslo, crear una técnica para llegar al mismo resultado, hacer un ordenamiento de valores (...) porque es un estudiante que no solo emplea algoritmos, ocupa mucho más su cabeza que el otro, porque hay mucho más razonamiento y pensamiento en este estudiante". (Profesor, 1. p. 8).

Los dos atributos mencionados hasta el momento, apuntan hacia la elaboración de un EBRAM, que corresponde a un estudiante que posee una habilidad y una inteligencia lógica-matemática (racionalidad) superior al del resto de sus compañeros. De lo anterior se deduce que desde la perspectiva de los docentes esto implicaría que un EBRAM posee también un nivel inteligencia (entendida como coeficiente intelectual) más elevado que el de sus pares (Cerda et al., 2011; Llewelyn, 2012; Mönks y Mason, 2000). 
En conjunto con la habilidad matemática y la racionalidad se puede mencionar la importancia que tiene la autorregulación de conductas para los docentes como requisito para que un estudiante pueda ser considerado como EBRAM. Dentro de las conductas legitimadas por los profesores en este ámbito se encuentran: el orden, la puntualidad y la rapidez en la ejecución de los cálculos. De esta manera lo propone una educadora reafirmando lo mencionado con anterioridad:

"Que tenga buena asistencia, que sea puntual, que tú, lo que pidas que haga, lo haga y si puede más, más aún, eso (...) y Ahh! que sea ordenado, yo creo si tiene que haber un orden, como en todas las cosas debe haber un orden". (Profesor 2, p. 4).

Es necesario mencionar en este punto que es primordial para los docentes que un EBRAM sea eficiente en la ejecución de los cálculos que impliquen aplicar algoritmos elementales de la forma más rápida que sea posible. Esta característica se encuentra vinculada a las exigencias que plantea el MINEDUC (2012) en términos del logro de una cobertura curricular completa, lo que implica, de acuerdo a los educadores entrevistados, "pasar mucha materia" en un tiempo muy acotado. Es así que lo pone de manifiesto una docente: "El MINEDUC, con la cantidad de contenidos y la cobertura curricular que busca, yo creo que busca un estudiante mecanizado, que responda rápido, que sea eficiente" (Profesor 3, p. 9).

Como último atributo presente en la construcción del EBRAM se encuentra la familia. En las narrativas de los docentes se visualiza un gran valor asignado al núcleo familiar. Específicamente, se hace mención a que un EBRAM debe poseer una "familia bien constituida", entendiendo a esta como aquella en la cual están presentes ambos progenitores, quienes se encargan de prestar todo el apoyo educativo que busque complementar la labor de la escuela (Paz, 2007). De esta forma lo propone un educador entrevistado:

"Un EBRAM debe ejercitar y bueno tener un apoyo familiar, tener una familia bien constituida y todo eso va a llevar a que un niño pueda ser exitoso cognitivamente y ser un buen estudiante de matemática, situación que no ocurre cuando las familias son disfuncionales". (Profesor 1, p. 4).

Respecto a este último atributo que emergió desde las narrativas docentes, existe la construcción de un vínculo entre los estudiantes que manifiestan un bajo rendimiento en la matemática escolar y las familias que han sido designadas como aquellas que no están "bien constituidas". Precisamente estas últimas serían caracterizadas como aquellas en las que existen múltiples problemas asociados a situaciones de precariedad socioeconómica y de diversas situaciones que exponen a los integrantes de estas en un permanente riesgo social, como también aquellas en las cuales solo un progenitor es el jefe de la familia, es decir, las familias monoparentales (Jadue, 2003; Paz, 2007).

Para este caso en particular, las familias se constituyen, a juicio de los docentes, en un factor limitante y a la vez excluyente para que un niño o joven pueda ingresar a la categoría de EBRAM. Es más, tienden a realizar la asociación entre lo que ellos denominan "familia disfuncional" con un bajo rendimiento en matemáticas. Así lo deja de manifiesto un profesor al ser consultado al respecto:

\footnotetext{
“A los niños que más les cuesta la matemática son los que menos apoyo tienen familiarmente,
} son los niños que prácticamente están solos durante el día, sus papás trabajan mucho, 
o viven solos con la mamá y la mamá trabaja o hay muchos niños que sus papás son alcohólicos o drogadictos (...) el problema son las familias mal constituidas o disfuncionales" (Profesor 1, p. 12).

\subsection{LA FORMACIÓN DE LAS ELECCIONES ESTRATÉGICAS: LOS SABERES DE LA MATEMÁTICA ESCOLAR QUE EL EBRAM DEBE MANIFESTAR}

Los saberes que favorecen y legitiman los docentes participantes en esta investigación son aquellos que propone el MINEDUC a través de las Bases Curriculares en Educación Matemática del año 2012 y los Ajustes Curriculares del año 2009, respectivamente. Así también se puede observar en las narrativas de los profesores que existen saberes dentro de la matemática escolar que por su carácter instrumental serían indispensables para estos. Los saberes mencionados corresponden a las cuatro operaciones básicas de la aritmética.

En cuanto a la relevancia y el carácter de obligatoriedad que poseen los saberes propuestos por el MINEDUC, desde las narrativas de los docentes se propone una imposición de estos respecto a otros saberes matemáticos. La principal razón de la hegemonía de esta matemática curricular se debe a que es esta la que se va a medir por medio de SIMCE, lo que implica a su vez evaluar el profesionalismo del docente. De esta forma lo plasma uno de los educadores: "A ver, de acuerdo a las bases curriculares del MINEDUC lo que tú necesitas pasarle, lo que dicen los programas de estudio, él debería estar preparado para rendir una prueba, rendirla en óptimas condiciones, como por ejemplo el SIMCE" (Profesor 2, p. 8).

Tal como ya se mencionaba, existe consenso entre los profesores respecto a la importancia de las operaciones básicas de la aritmética. Para que un estudiante sea catalogado como un EBRAM debe manifestar el conocimiento de la correcta ejecución de los algoritmos asociados a las cuatro operaciones básicas. En este sentido, uno de los docentes propone:

"Las cuatro operaciones básicas las deben manejar, tienen que manejar las propiedades de, de lo que estemos trabajando específicamente, y llevar esa teoría a la práctica, sabiendo llevar esa teoría a la práctica siento yo que van a tener un buen resultado". (Profesor 1, p. 12).

Esta forma de legitimar a estos saberes como elementales o básicos por parte de los docentes es reforzada en las narrativas de los estudiantes, quienes señalan la importancia del conocimiento de las cuatro operaciones básicas, lo que queda en evidencia en el siguiente fragmento: "Primero, lo más principal es saberse los números también, saber sumar y restar, porque con la suma, la resta, la multiplicación, la división, entonces se necesita eso, es lo principal" (Estudiante 2, p. 2).

Por tanto, se puede visibilizar que los saberes de la matemática escolar que propone el MINEDUC son los legitimados por los docentes dentro del aula. Además, estos son considerados como relevantes e imprescindibles por el hecho de que son evaluados por el SIMCE, lo que valida que sean estos saberes y no otros los que son reconocidos como legítimos por los profesores en esta escuela. Los supuestos que hay detrás de estas creencias se basan en la supuesta naturaleza objetivable y medible de estos conocimientos, como también por importancia en cuanto cumplen una función evaluadora, bajo la lógica premio-castigo hacia alumnos y profesores. Bajo este "régimen de verdad" es que logran un estatuto de saberes verdaderos e incuestionables dentro del aula (Popkewitz, 2013). 
Estudios Pedagógicos XLII, N 3: 223-241, 2016

ANÁLISIS CRITICO DEL DISCURSO DE PROFESORES DE MATEMÁTICAS Y SUS ESTUDIANTES: SUBJETIVIDADES

Y SABERES EN AULAS HETEROGÉNEAS

Lo anterior demuestra la existencia en este contexto de una enseñanza de la matemática con una finalidad instrumental, lo que se asocia con una enseñanza de esta asignatura más tradicional, platónica y estructural, es decir, en palabras de Cantoral et al., (2014):

[...] aquella matemática que se centra en los objetos matemáticos, entidades abstractas que son ejemplificadas y ejercitadas [...] esto implica que las matemáticas tratan con objetos abstractos, anteriores por tanto a la praxis social, externas al individuo, consideradas como verdades preexistentes. (p. 18).

No obstante, se observa que existe por parte de los docentes una disposición parcial por integrar en sus clases, específicamente al inicio de las unidades didácticas y a modo de ilustración, los saberes matemáticos de los estudiantes que emplean en otros contextos, lo que se ha denominado desde la literatura como la "matemática de la calle" (D'Amore, 2000, p. 324). Esta última es entendida como aquellos saberes de las matemáticas que son empleados generalmente en contextos externos a la institución escolar, y que se vinculan a prácticas sociales tales como las ventas que realiza un vendedor ambulante, o las que lleva a cabo un niño que trabaja vendiendo en un mercado o feria libre (Nuñes y Saxe 1990, citados en D’Amore, 2000).

En la misma línea argumentativa, en el inicio de las unidades didácticas los saberes de las matemáticas de los estudiantes sirven a los profesores solamente para ilustrar los contenidos de esta asignatura, lo que los docentes denominan como la contextualización de los conocimientos de la matemática. De esta forma lo asevera una educadora:

"Uno parte la unidad preguntando, contextualizándolo en el inicio, por ejemplo, hoy día vamos a presentar los números enteros ¿Dónde observamos los número enteros? [...] y a a partir de la tercera clase, se te olvida el contexto, porque el programa te pide que debes llegar a división y a resolución de problemas". (Profesora 3, p. 4).

A la vez, es posible visualizar que los saberes de la matemática de la calle de los estudiantes son relegados a un plano inferior respecto a los saberes de la matemática escolar propuestos por el MINEDUC, y que son legitimados por medio de los discursos de los docentes. Para ilustrar lo aseverado, se observó en las entrevistas individuales aplicadas a los estudiantes que todos los alumnos lograron dar respuesta a aquel problema matemático que a juicio de ellos correspondía a una situación más cercana a sus experiencias cotidianas de uso de las matemáticas, lo que confirma el hecho de que para los sujetos escolares es más significativo el uso de esta asignatura asociada a un contexto particular, a una necesidad específica, es decir, a un uso situado de las matemáticas con un claro referente contextual (Cantoral et al., 2014; Knijnik, 2007; Walkerdine 1997 Cit. en Llewelyn, 2012).

Para el caso de los estudiantes entrevistados, se logró plasmar en sus narrativas que se sienten más seguros y cómodos con el uso de las matemáticas en otros contextos distintos al aula:

"Yo creo que soy mejor con la matemática, más cuando voy comprar. Sí porque son cosas simples. Porque tu pagai y sacai la cuenta y así y sacai rápido las cuentas, mientras que aquí te piden cosas fracciones y cosas así, pero esas cosas no po', porque tú llegai y pagai y tenis que calcular cuánto te van a dar de vuelto". (Estudiante 2, p. 6). 


\subsection{GUBERNAMENTALIDAD: EFECTOS DE LOS DISCURSOS DOCENTES DE MATEMÁTICAS EN LA CONSTITUCIÓN DE LAS SUBJETIVIDADES DE SUS ESTUDIANTES}

En este acápite se pretende visibilizar cómo se conducen, guían y orientan, desde los discursos de profesores y estudiantes, formas particulares de la construcción del "yo" por parte de los alumnos, de forma tal de identificar aquellas narrativas en donde se favorezcan atributos que incidan en la constitución de subjetividades, las que pueden acercarse o, por contraste, alejarse de la noción conceptual del EBRAM.

Uno de los aspectos que más llama la atención es la forma en la cual los profesores identifican tensiones que los obligan a promover ciertas características en sus estudiantes, que a juicio de los docentes responden a la lógica implícita en los programas de estudio de matemática escolar que propone el MINEDUC, es, pues, en este sentido, que uno de los educadores menciona: "El MINEDUC con la cantidad de contenidos y la cobertura curricular que busca, yo creo que busca un estudiante mecanizado, que responda rápido, que sea eficiente" (Profesor 3, p. 9).

Por tanto, los profesores interpretan que son interpelados por el saber poder de las políticas educativas del MINEDUC, para formar estudiantes que en el área de matemática escolar sean capaces de responder una evaluación estandarizada de manera eficiente, solo aplicando cálculos y empleando saberes matemáticos básicos, llevando a la mecanización de los procesos de aprendizaje de las matemáticas por parte de los educandos (Cantoral et al., 2014; Skovsmose et al., 2008). En este sentido, existe también un rechazo parcial por parte de uno de los docentes a reproducir dichos discursos. De esta forma uno de los educadores asevera:

"Eso es lo que te piden y eso es lo que se valora, lo que te está pidiendo el sistema de educación chileno y yo no estoy para crear ese tipo de niños, yo quiero crear niños críticos y yo quiero crear niños que sean capaces de pensar y que no se basen, como pasa en Chile, en la nota". (Profesor 1, p. 9).

A pesar de observarse una parcial "resistencia" por parte de los profesores hacia la formación de un estudiante mecanizado, competitivo y eficiente en matemática escolar, se puede constatar contradicciones en las narrativas de los educadores, ya que estos terminan por favorecer muchos de aquellos atributos mencionados en la elaboración discursiva del EBRAM (habilidades matemáticas, racionalidad, eficiencia, manejo de las cuatro operaciones básicas, proactividad, etc.) y que están alineados con lo que ellos deducen que propone el MINEDUC en la lógica del accountability o la rendición de cuentas por medio de resultados (Falabella, 2014).

Así también se pueden evidenciar cómo, finalmente, el discurso se materializa en las prácticas pedagógicas que los docentes implementan en el cotidiano. En este sentido, los educadores favorecen clases más centradas en la repetición y ejecución de ejercicios rutinarios, reproduciendo la lógica de la enseñanza de una matemática escolar más tradicional o platónica (Cantoral et al., 2014). A modo de ilustración, una de las docentes entrevistadas declara: "Entonces, siempre, por ejemplo, en los sextos que estoy con ellos, siempre parto con una pregunta SIMCE, ya la analizamos, les doy un tiempo para que la desarrollen" (Profesora, 2, p. 3). 
Estudios Pedagógicos XLII, N 3: 223-241, 2016

ANÁLISIS CRIITICO DEL DISCURSO DE PROFESORES DE MATEMÁTICAS Y SUS ESTUDIANTES: SUBJETIVIDADES Y SABERES EN AULAS HETEROGÉNEAS

Es posible constatar, entonces, que si bien existe consciencia por parte de los profesores respecto a que no hay una intención explícita de reproducir y formar un "estudiante mecanizado" centrado en la obtención de buenas calificaciones y en lograr un excelente puntaje en el SIMCE, a pesar de ello, los docentes favorecen en sus prácticas discursivas atributos que están relacionados con la constitución de un EBRAM y que están a su vez alineadas con la lógicas asociadas a considerar al aprendizaje escolar como un dispositivo de control, lo que obedece a procesos de rendición de cuentas o accountability en donde se busca optimizar los resultados en un mínimo de tiempo, objetivos educativos en donde la matemática escolar se puede transformar en una excelente aliada (Falabella, 2014).

Otro aspecto central en el cual se puede visibilizar la forma en la cual los discursos de los docentes conducen las subjetividades de los estudiantes se puede evidenciar en la manera en que estos persiguen que sus alumnos se autorregulen, de manera más específica, en la relevancia que le otorgan a la atención como una conducta privilegiada y fundamental para el aprendizaje de la matemática (Popkewitz, 2013).

Es tan patente este efecto que son los propios alumnos los encargados de internalizar este tipo de control externo, y, con ello, ser capaces de mantenerse atentos en las clases de matemática, específicamente focalizar su atención en las dinámicas de trabajo grupal, en donde evitan distraerse, ya que asumen que los discursos pedagógicos en este aspecto tienen legitimidad, por tanto son verdaderos, de tal manera que ellos mismos son los que se autorregulan y, en caso contrario, se culpan cuando son conscientes de que han estado distraídos en clases. Así lo expone uno de los estudiantes entrevistados:

"A veces cuando las tareas son en grupo, en grupo, pero el profe quiere que sea individual, porque yo creo que el profe quiere que uno aprenda, porque cuando uno está con el amigo, como que uno se desconcentra y empiezan a ser puras cosas, y empiezan a distraerse entre ellos mismos". (Estudiante 2, p. 4).

En este contexto educativo en particular, se visibilizó en las narrativas que los educadores favorecen ciertas conductas que tienden a manifestarse por los estudiantes que han sido denominados como EBRAM. Este modelamiento de conductas, por medio del control externo que ejercen los docentes, pasa a transformarse y mutar en un control interno por parte del joven, constituyéndose la autorregulación de la conducta $-\mathrm{y}$ en este caso en particular la atención específicamente- en una tecnología del yo, es decir, en una forma de autogobierno y perfeccionamiento de sí mismo (Larrosa, 1995; Rose, 1999; Veiga-Neto, 2010).

Por lo tanto, la responsabilidad de autorregularse y controlarse es trasladada hacia el estudiante, quien debe internalizar aquellas conductas que favorecen su aprendizaje de la matemática escolar, conductas que son coherentes con aquellos atributos que constituyen al EBRAM. En otras palabras, el sujeto escolar se convierte a la vez en un buen actor y en un empresario de sí mismo, en gestor de sus aprendizajes y de su comportamiento (Gómez y Jódar, 2007; Grinberg, 2006).

Además, es posible detectar en las narrativas de los alumnos que se alejan de la construcción normativa denominada EBRAM el efecto de atribuirse ellos mismos el apelativo de "no ser buenos para la matemática". Ante este hecho, estos educandos tienden a asociar directamente que esta etiqueta les ha sido asignada, debido a la falta de un atributo o, en particular, a la ausencia de la habilidad matemática. Esta tendencia se puede visibilizar 
en el caso de un alumno que declara que su bajo rendimiento en matemática escolar tiene explicación al examinar su historia familiar: "Porque siempre me ha costado. En realidad no solo a mí, a toda a mi familia como que igual a todos les cuesta dividir, y no encuentro que sea solo de mi parte, viene de mi familia" (Estudiante 1, p. 2).

Se puede dar cuenta entonces que tanto para el estudiante que ha sido categorizado como EBRAM como para aquel que no corresponde a dicha etiqueta se presencian discursos y conductas coherentes con el nombre categorial que les ha sido asignado. Es decir, ambos "tipos" de alumnos tienden a performar (en el sentido butleriano) el rol que se les ha impuesto discursivamente; en otras palabras, a internalizar dicho nombre y comportarse en consecuencia al rótulo por el cual son convocados dentro de esta institución educativa (Gregson y Rose, 2000).

\section{DISCUSIÓN FINAL}

A partir del análisis que se llevó a cabo en esta investigación se evidencio en cuanto a la construcción discursiva del EBRAM que los atributos que lo constituyen corresponden a la posesión de una habilidad matemática innata, ser un sujeto racional, la autorregulación de sus conductas y la pertenencia a una familia bien constituida. Esta noción de EBRAM caracterizada con anterioridad, emerge, se construye y se legitima a través de los discursos de docentes y estudiantes en esta institución educativa, noción que genera en sí una serie de tensiones y problemáticas para los agentes educativos involucrados en esta investigación, que son necesarias de analizar y discutir; problemáticas en las que se centrará brevemente esta discusión final.

Por una parte, se puede mencionar la problemática que surge a partir de la elaboración discursiva de un estudiante norma, el cual emerge producto de un sistema de clasificación, categorización y jerarquización de alumnos que se basa en las directrices presentes en las políticas educativas, el currículum de educación matemática y en los documentos que orientan la construcción de pruebas estandarizadas como PISA a nivel internacional o SIMCE a nivel local (Lewellyn, 2012; Popkewitz, 2013). Siguiendo a Popkewitz (2013), son estas evaluaciones las que por medio de su saber poder promueven un tipo de particular de subjetividad en los estudiantes, suponiendo la existencia de un alumno promedio, como si fuese un hecho real, quien es capaz de demostrar saberes matemáticos que han sido estandarizados para su medición y control.

Lo anteriormente expuesto se constituye en una problemática debido a que en el contexto en donde se llevó a cabo esta investigación, la mayoría de los estudiantes, de acuerdo a las narrativas de los participantes de este estudio, no cumplen con los atributos que los calificarían para ingresar a la categoría de EBRAM. Esto debido a que los marcadores de subjetividad que constituyen a este estándar distan respecto a la forma en la cual son razonados los sujetos escolares que asisten a este centro educativo y a este tipo de establecimientos educacionales (municipales), educandos que por los demás son caracterizados como niños o jóvenes pertenecientes a las minorías, niños vulnerables y deficitarios en cuanto a su capacidad de aprendizaje (Infante et al., 2011). Específicamente, estos jóvenes son catalogados como carentes de la habilidad matemática innata que les facilitaría lograr el éxito académico en esta asignatura escolar. Estas formas de entender a los estudiantes corresponden a las denominadas "practicas divisorias" asociadas con la 
Estudios Pedagógicos XLII, N 3: 223-241, 2016

ANÁLISIS CRIITICO DEL DISCURSO DE PROFESORES DE MATEMÁTICAS Y SUS ESTUDIANTES: SUBJETIVIDADES

Y SABERES EN AULAS HETEROGÉNEAS

creación de grupos y categorías de alumnos por parte de los docentes, acciones vinculadas a la objetivación de los alumnos, a los que se les asigna una etiqueta para hacerlos legibles y a la vez objetos de medición y control (Ball, 1997).

Estos sistemas para razonar a los estudiantes terminarían por determinar las subjetividades de los alumnos a solo unos cuantos atributos, fijando sus identidades a una construcción conceptual estática que no responde a la heterogeneidad de marcadores de subjetividad y a los saberes matemáticos que los alumnos ponen en juego en sus prácticas diarias con el uso de las matemáticas (Cantoral et al., 2014; Stentotf y Valero, 2009).

En la misma línea argumentativa, al construir un estudiante "norma" discursivamente, hay que evidenciar también los efectos que tiene esta construcción en los alumnos, que para este caso corresponde a la función que cumple la gubernamentalidad en este análisis. En esta investigación se visibilizó que los estudiantes tienden a performar (en el sentido butleriano) la etiqueta que les ha sido asignada, tanto para quienes responden a la categoría de EBRAM como también para aquellos que no son convocados por este rótulo. En el caso de los estudiantes que son interpelados por la etiqueta de EBRAM, estos tienden a internalizar el discurso de los docentes que así los nomina. Es, en este sentido, que estos alumnos se transforman en gerentes de sí mismos, haciéndose responsables de sus aprendizajes, de gestionar sus conductas y habilidades, con la finalidad de capitalizar sus posibilidades educativas al máximo (Gómez y Jodar, 2007; Rose, 1999). Lo que es coherente bajo la lógica de un sistema educativo como el chileno, en donde en este cuasi mercado educativo dominado por las lógicas neoliberales es el sujeto quien tiene que responsabilizarse por su actuar, es decir, es el único responsable de sus acciones, y, por tanto, si no logra el éxito, los sentimientos de culpa y aflicción afloran de manera espontánea.

Como última reflexión vinculada con la gubernamentalidad, se puede mencionar la función productora de subjetividades implícita en el proceso de enseñanza-aprendizaje de la matemática escolar. Generalmente, desde la didáctica se propone el esquema denominado “triángulo didáctico", por medio del cual se describen las relaciones entre el saber, el docente y el estudiante en el proceso de enseñanza-aprendizaje de una asignatura cualquiera ( $D^{\prime}$ Amore, 2000). En ninguna de las interacciones presentes que contempla este modelo teórico se considera la constitución de las subjetividades de docentes y alumnos, como también no se problematizan las relaciones de saber poder de los saberes de la matemática escolar prescrita por un currículum, los que se legitiman como "el régimen de verdad" que deben consensuar tanto maestros como aprendices. Por ello, es clave ampliar la visión estática y restringida del proceso de aprendizaje de la matemática escolar desde un enfoque dinámico que considere las relaciones de poder y de constitución de subjetividades entre los vértices que componen al ya mencionado triángulo didáctico (Larrosa, 1995; Popkewitz, 2004).

Es el presente estudio un esfuerzo que se centró en escudriñar un fenómeno muy complejo desde una perspectiva de análisis diferente, la que también buscó proveer de las herramientas teóricas y metodológicas para seguir avanzando en la investigación desde esta perspectiva, la que propone problematizar, y con ello iluminar aquellas aristas del objeto de estudio que han sido poco exploradas y que precisan de una profundización para cuestionar y también ayudar a enriquecer el análisis de aquellos enfoques que circunscriben sus explicaciones solamente en los sujetos (estudiantes y profesores) y no en las estructuras que sirven de soporte de los discursos que legitiman una forma estática y restringida de razonar al estudiante que ha sido entendido como EBRAM, o, por el contrario, como un alumno con dificultades de aprendizaje en la matemática escolar. 


\section{REFERENCIAS BIBLIOGRÁFICAS}

Agencia de la Calidad de la Educación (2014). Resultados nacionales SIMCE 2013. Recuperado de http://www.agenciaeducacion.cl/resultados-nacionales-simce-2013/

Aravena, M., \& Caamaño, C. (2007). Modelización matemática con estudiantes de secundaria de la comuna de Talca, Chile. Estudios Pedagógicos, 33(2). Recuperado de http://www.scielo.cl/ scielo.php?pid=S071807052007000200001\&script=sci_arttext

Ball, S. (1997). Foucault y la educación. Disciplinas y saber. Madrid: Ediciones Morata.

Bellei, C., Múñoz, G., Pérez, L., \& Raczynski, D. (2004). ¿Quién dijo que no se puede? Escuelas Efectivas en Sectores de Pobreza. Santiago de Chile: UNICEF.

Banco Interamericano de Desarrollo. (2010). La condición de la educación en matemáticas y ciencias naturales en América Latina y el Caribe. División de Educación. Recuperado de http:// www.iadb.org/es/temas/educacion/bid-ensenanza-de-ciencias-y-matematicas-en-americalatina,6494.html

Canales, J. (2012). La Arqueología del Saber de Michel Foucault o la caja de herramientas: un análisis enunciativo de resistencia a los dispositivos (Tesis de Licenciatura). Universidad de Chile, Santiago. Recuperado de http://tesis.uchile.cl/bitstream/handle/2250/111480/Canales\%20 Javiera.pdf?sequence $=1$

Cantoral, R., Reyes-Gasperini, D., \& Montiel, G. (2014). Socioepistemología, Matemáticas y Realidad. Revista Latinoamericana de Etnomatemáticas, 7(3), 91-116. Recuperado de http:// www.revista.etnomatematica.org/index.php/RLE/article/view/149/161

Casassus, J. (2010). Las Reformas basadas en estándares: un camino equivocado. Revista Educere et Educare, 5(9), 85-107. Recuperado de http://www.erevistas.csic.es/ficha_articulo. php?url=oai:ojs.erevista.unioeste.br:article/4082\&oai_iden=oai_revista993

Castro A., Mardones E., Ortiz, A., \& Quiroga, F. (2013). Interacciones alumnos-profesor respecto de la ocurrencia de episodios de violencia simbólica en clases de matemática el contexto de vulnerabilidad. Formación Universitaria, 6(1). Recuperado de http://www.scielo.cl/scielo. php?pid=S0718-50062013000100005\&script=sci_arttext

Cerda, G., Flores, C., Melipillán, R., Ortega, R., \& Pérez, C. (2011). Inteligencia lógica y rendimiento académico en matemáticas: un estudio con estudiantes de Educación Básica y secundaria en Chile. Anales de Psicología, 27(2), 389-398. Recuperado de http://revistas.um.es/analesps/ article/download/123011/115641

Creswell, J. (2007). Qualitative inquire and research design: choosing among five approaches. Nebraska: Sage Publications.

D’Amore, B. (2000). Escolarización del saber y de las relaciones: efectos sobre el aprendizaje de las matemáticas. Revista Latinoamericana de Investigación en Matemática Educativa, 3(3), 321337. Recuperado de http://www.redalyc.org/pdf/335/33503304.pdf

Donoso, S., \& Hawes, G. (2002). Eficiencia escolar y diferencias socioeconómicas: a propósito de los resultados de las pruebas de medición de la calidad de la educación en Chile. Educaçao e pesquisa, 28(2), 25-39. Recuperado de http://www.scielo.br/pdf/ep/v28n2/a03v28n2.pdf

Espinoza, O. (2012). Arqueología del discurso de las competencias en la formación inicial docente en Chile (Tesis de Doctorado). Pontificia Universidad Católica de Chile, Santiago.

Falabella, A. (2014). The performing school. The effects of market \& accountability policies. Education Policy Analysis Archives, 22(70). Recuperado de http://epaa.asu.edu/ojs/article/ view/1315

Foucault, M. (2013). La arqueología del saber. Buenos Aires: Grupo Editorial Siglo XXI.

Gómez, L., \& Jódar, F. (2007). Educación Posdisciplinaria, formación de nuevas subjetividades y gubernamentalidad neoliberal. Herramientas conceptuales para el análisis del presente. Revista Mexicana de Investigación Educativa, 12(32), 381-404. Recuperado de http://www.redalyc.org/ articulo.oa?id=14003218 
Estudios Pedagógicos XLII, N 3: 223-241, 2016

ANÁLISIS CRITICO DEL DISCURSO DE PROFESORES DE MATEMÁTICAS Y SUS ESTUDIANTES: SUBJETIVIDADES

Y SABERES EN AULAS HETEROGÉNEAS

Gregson, N., \& Rose, G. (2000). Taking Butler elsewhere: performativities, spatialities and subjectivities. Environment and Planning D: Society and Space, 18(4), 433-452. doi:10.1068/ d232

Grinberg, S. (2006). Educación y gubernamentalidad en las sociedades de gerenciamiento. Revista Argentina de Sociología, 4(6), 67-87.

Hernández, D. (2010). Arqueología del saber y orden del discurso: un comentario sobre las formaciones discursivas. En-claves del pensamiento, 4(7), 47-61. Recuperado de http://www. scielo.org.mx/scielo.php?script=sci_arttext\&pid=S1870-879X2010000100003

Infante, M. (2010). Desafíos para la formación docente: Inclusión educativa. Estudios Pedagógicos, 36(1), 287-297. Recuperado de http://www.scielo.cl/scielo.php?pid=S071807052010000100016\&script=sci_arttext

Infante, M., Matus, C., \& Vizcarra, R. (2011). Razonando sobre la idea de diferencia en las políticas educativas chilenas. Universum, 2(2), 143-163. Recuperado de http://www.scielo.cl/scielo. php?pid=S0718-23762011000200008\&script=sci_arttext

Jadue, G. (2003). Transformaciones familiares en Chile: Riesgo creciente para el desarrollo emocional, psicosocial y la educación de los hijos. Estudios Pedagógicos, 29(1), 115-126. Recuperado de http://www.scielo.cl/scielo.php?pid=S0718-07052003000100008\&script=sci_arttext

Jäger, S. (2008). Entre las culturas: caminos fronterizos en el análisis del discurso. Pensar la relación Análisis Crítico del Discurso y Educación. Discurso \& Sociedad, 2(3), 503-532. Recuperado de http://www.dissoc.org/ediciones/v02n03/DS2\%283\%29Jaeger.html

Kanes, C., Morgan, C., \& Tsatsaroni, A. (2014). The PISA mathematics regime: knowledge structures and practices of self. Educational Studies in Mathematics, 87, 145-165. doi:10.1007/s10649014-9542-6

Knijnik, G. (2007). Diversidad cultural, matemáticas y exclusión: oralidad y escritura en la educación matemática campesina en el sur de Brasil. En J. Giménez, J. Diez-Paloma, y M. Civil (Coords.), Educación Matemática y Exclusión (pp. 63-82). Barcelona: Grao.

Larrosa, J. (1995). Escuela, Poder y Subjetivación. Madrid: La Piqueta.

Llewelyn, A. (2012). Unpacking understanding: the (re)search for the Holy Grail of mathematics education. Educational Studies in Mathematics, 81, 385-399. doi:10.1007/s10649-012-9409-7

Matus, C. (2005). ¿Existe alguna posibilidad de que triunfe la diversidad? Revista Pensamiento Educativo, 37(2), 16-26. Recuperado de http://pensamientoeducativo.uc.cl/index.php/pel/ article/view/352/754

MINEDUC. (2009). Objetivos Fundamentales y Contenidos Mínimos Obligatorios de la Educación Básica y Media, Actualización 2009. Autor. Recuperado de www.curriculumenlinea.mineduc. cl/descargar.php?id_doc

MINEDUC. (2012). Bases curriculares de Educación Matemática. Autor. Recuperado de http:// curriculumenlinea.mineduc.cl/sphider/search.php?query\&t_busca=1\&results\&search=1\&dis= $0 \&$ category $=1$

Mönks, F., \& Mason, E. (2000). Developmental psychology and giftedness: theories and research. In K. Heller, F. Mönks, R. Sternberg, \& R. Subotnik (Eds.), International Handbook of Giftedness and Talent (pp. 124-198). Oxford: Pergamon Press.

Paz, S. (2007). Problemas en el desempeño escolar y su relación con el funcionalismo familiar en alumnos de EGB1. Revista Facultad de Medicina, 8(1), 27-32. Recuperado de http://www. fm.unt.edu.ar/Servicios/publicaciones/revistafacultad/vol_8_n_1_2007/cap5.pdf

Peña. M. (2013). Análisis crítico de discurso del decreto 170 de subvención diferenciada para Necesidades Educativas Especiales: El diagnóstico como herramienta de gestión. Revista Psicoperspectivas: Individuo y Sociedad, 12(2), 252-286. Recuperado de http://www. psicoperspectivas.cl/index.php/psicoperspectivas/article/view/252/286

Popkewitz, T. (2004). The Alchemy of the Mathematics Curriculum: Inscriptions and the Fabrication of the Child. American Educational Research Journal, 41(1), 3-34. 
doi: $10.3102 / 00028312041001003$

Popkewitz, T. (2013). PISA: Números, estandarización de la conducta y la alquimia de las materias escolares. Profesorado, Revista de Currículum y formación del profesorado, 17(2), 47-64. Recuperado de http://www.ugr.es/ recfpro/rev172ART3.pdf

Popkewitz, T., \& Brennan, M. (2000). El desafio de Foucault: Discurso, conocimiento y poder en la educación. Barcelona: Pomares-Corredor.

Román, M. (2011). ¿Por qué los docentes no pueden desarrollar procesos de enseñanza aprendizaje de calidad en contextos sociales vulnerables? Persona y Sociedad, 17(1), 113-128. Recuperado de http://www.personaysociedad.cl/\%C2\%BFpor-que-los-docentes-no-pueden-desarrollarprocesos-de-ensenanza-aprendizaje-de-calidad-en-contextos-sociales-vulnerables/

Rose, N. (1999). Governing the soul: the shaping of the private self. London: Free Associations Books.

Ruíz, J. (2003). Metodología de la Investigación cualitativa. Deusto: Universidad de Deusto.

Skovsmose, O., Alro, H., \& Valero, P. (2008). Antes de dividir, se tiene que sumar. Entrevistar porvenires de estudiantes indígenas. Revista Latinoamericana de Etnomatemáticas, 1(2), 111-136. Recuperado de http://www.etnomatematica.org/v1-n2-julio2008/Valero-Skovsmose-Alro.pdf

Stentoft, D., \& Valero, P. (2009). Identities-in-action: exploring the fragility of discourse and identity in learning mathematics. Nordic Studies in Mathematics Education, 14(3), 55-77. Recuperado de http://www.smerg.moodle.ell.aau.dk/file.php/1/Notes/SMERG_Meeting_12-1-10/055078_ stentoft.pdf

Veiga-Neto, A. (2010). Gubernamentalidad neoliberal: implicaciones para la educación. Revista Educación y Pedagogía, 22(58), 213-235. Recuperado de http://aprendeenlinea.udea.edu.co/ revistas/index.php/revistaeyp/article/view/9748/8965

Youdell, D. (2010). School Trouble: identity, power and politics in education. Abingdon: Routledge. 
